\title{
YbGaGe: normal thermal expansion
}

\author{
Y. Janssen ${ }^{\mathrm{a}, *}$ S. Chang ${ }^{\mathrm{a}}$ B.K. Cho ${ }^{\mathrm{a}, \mathrm{b}}$ A. Llobet ${ }^{\mathrm{c}}$ K.W. Dennis ${ }^{\mathrm{a}}$ \\ R.W. McCallum ${ }^{\mathrm{a}}$ R.J. Mc Queeney ${ }^{\mathrm{a}, \mathrm{d}}$ P.C. Canfield ${ }^{\mathrm{a}, \mathrm{d}}$ \\ a Ames Laboratory, 50011 Ames IA \\ ${ }^{\mathrm{b}}$ Center for Frontier Materials and Department of Materials Science and \\ Engineering, GIST, Kwangju 500-712, South Korea \\ ${ }^{\mathrm{c}}$ Los Alamos National Laboratory, 90120 Los Alamos, NM \\ ${ }^{\mathrm{d} D e p a r t m e n t}$ of Physics and Astronomy, Iowa State University, 50011 Ames IA
}

\begin{abstract}
We report evidence of the absence of zero thermal expansion in well-characterized high-quality polycrystalline samples of YbGaGe. High-quality samples of YbGaGe were produced from high-purity starting elements and were extensively characterized using x-ray powder diffraction, differential thermal analysis, atomic emission spectroscopy, magnetization, and neutron powder diffraction at various temperatures. Our sample melts congruently at $920^{\circ} \mathrm{C}$. A small amount of $\mathrm{Yb}_{2} \mathrm{O}_{3}$ was found in our sample, which explains the behavior of the magnetic susceptibility. These observations rule out the scenario of electronic valence driven thermal expansion in YbGaGe. Our studies indicate that the thermal expansion of YbGaGe is comparable to that of $\mathrm{Cu}$.
\end{abstract}

Key words: YbGaGe, intermetallics, neutron diffraction, magnetic measurements, thermal analysis

\section{Introduction}

Recently, it was reported that the compound YbGaGe shows negligible thermal expansion between 100 and $400 \mathrm{~K}$ [1]. This effect was attributed to a progressive change in the $\mathrm{Yb}$ electronic valence. It was also noted that the exact composition is crucial, since for some of the $\mathrm{YbGa}_{1+x} \mathrm{Ge}_{1-x}$ the zero

\footnotetext{
* Corresponding author.

Email address: yjanssen@ameslab.gov (Y. Janssen).
}

Preprint submitted to Journal of Alloys and Compounds 3 September 2018 
thermal expansion occurs, whereas negative and positive thermal expansion also occur, presumably for other values of $x$. However, the precise values for $x$ were not reported. In a more recent publication by the same authors [2], the compound with composition $\mathrm{YbGa}_{1.05} \mathrm{Ge}_{0.95}$ was reported to have a sudden valence transition at $5 \mathrm{~K}$, accompanied by a large change in the lattice parameters, as determined by high-resolution X-ray powder diffraction.

Most materials having negative thermal expansion (NTE) are insulating oxides, where the NTE effect is caused by an underconstrained crystal lattice [3]. The number of metals possessing NTE are few, with the most notable case being the Invar alloys. In the metallic NTE materials, usually two local atomic configurations with different atomic sizes exist, with the smaller sized atom being an excited state. These atomic configurations can be different magnetic (Invar) and/or valence states. Delta-plutonium is a possible example of an NTE material caused by a valence transition and has received considerable attention lately [4]. However, $\mathrm{Pu}$ is difficult to study, making YbGaGe a potentially very interesting material for the study of NTE behavior of electronic origin.

We have prepared single-phase polycrystalline YbGaGe by combining highpurity $\mathrm{Yb}, \mathrm{Ga}$ and $\mathrm{Ge}$, and characterized it by means of x-ray powder diffraction, chemical analysis, differential thermal analysis and measurements of magnetization. We also performed neutron powder diffraction in order to measure the temperature dependence of the lattice parameters, to further check the quality of the sample and to determine the distribution of the $\mathrm{Ga}$ and Ge ions within the lattice. Neutron diffraction was employed since Ga and Ge are nearly indistinguishable to standard x-ray diffraction techniques.

\section{Sample synthesis}

Salvador et al. [1] reported that they synthesized YbGaGe in the following, single-step, manner. They mention that the elements were directly combined in their stoichiometric ratios and heated to $850^{\circ} \mathrm{C}$ for 96 hours followed by a cool to room temperature over about 12 hours. The authors did not indicate the purity of their starting materials, nor did they mention the material of the crucibles that they used.

For the synthesis of our sample we used the high-purity elements $\mathrm{Yb}$ (Ames Laboratory, 99.94\% elements basis), Ga (Alfa Aesar, 99.999\% metals basis) and Ge (Alfa Aesar, 99.999\% metals basis). It is important to use elements of high-purity, because it is well known that impurities can stabilize or even enable the formation of intermetallic phases. Stoichiometric amounts, of liquid $\mathrm{Ga}$ and lumps of Ge and $\mathrm{Yb}$, with a total mass of about $8 \mathrm{~g}$, were put in a 
Ta crucible. We have found Ta crucibles to be inert for use with $\mathrm{Yb}, \mathrm{Ga}$ and $\mathrm{Ge}$ in the temperature range and composition of interest. To prevent evaporation of the volatile $\mathrm{Yb}$, the crucible was sealed by arc welding in an $\mathrm{Ar}$ atmosphere. The Ta crucible was placed in a silica ampoule that was evacuated and subsequently sealed.

The ampoule was placed in a furnace at room temperature. The furnace was heated to $200^{\circ} \mathrm{C}$ in 2 hours and, after that, to $850^{\circ} \mathrm{C}$ in 70 hours. The temperature was kept constant at $850^{\circ} \mathrm{C}$ for 40 hours, after which it was lowered to room temperature in 24 hours.

The sample came out of the Ta crucible easily and was brittle. However, the shapes of the $\mathrm{Yb}$ pieces were still discernible, suggesting that the formation of the desired phase was incomplete. A small amount, less than $0.5 \mathrm{~g}$, of the sample was finely ground and used for X-ray powder diffraction. The strongest reflections on the diffractogram could be indexed according to the reported crystal structure of YbGaGe [1], however, reflections belonging to undetermined secondary phases were clearly visible.

The remainder of the sample was coarsely ground and mixed for homogenization, and pressed into two pellets. These pellets were sealed in a new Ta crucible, that was sealed and put in a silica ampoule as described above. The ampoule was heated in a furnace up to $860^{\circ} \mathrm{C}$, and kept at this temperature for 10 days. After this, the ampoule was taken out of the furnace and allowed to cool down naturally.

After this second heat treatment, the pellets had their original shape, but the surfaces looked as though they had partially melted. This indicates the presence of a metallic liquid during formation. The sample was again brittle. $\mathrm{X}$-ray powder diffraction on different parts of the sample did not show any trace of any other phase besides YbGaGe. This X-ray single-phase sample was used for the experiments described below.

\section{Magnetization and thermal properties}

The temperature dependent magnetization of YbGaGe was measured in a Quantum Design Magnetic Properties Measurement System on a bulk piece of approximately $300 \mathrm{mg}$. Particular care was taken in the measurement of this low-moment sample, as extremely small quantities of ferromagnetic impurities or the sample holder may produce a small zero offset which can be independent of temperature. If no correction is made, an offset will give the appearance of large deviations from Curie-Weiss behavior at high temperatures, suggesting a temperature dependent magnetic moment. Therefore measurements were 


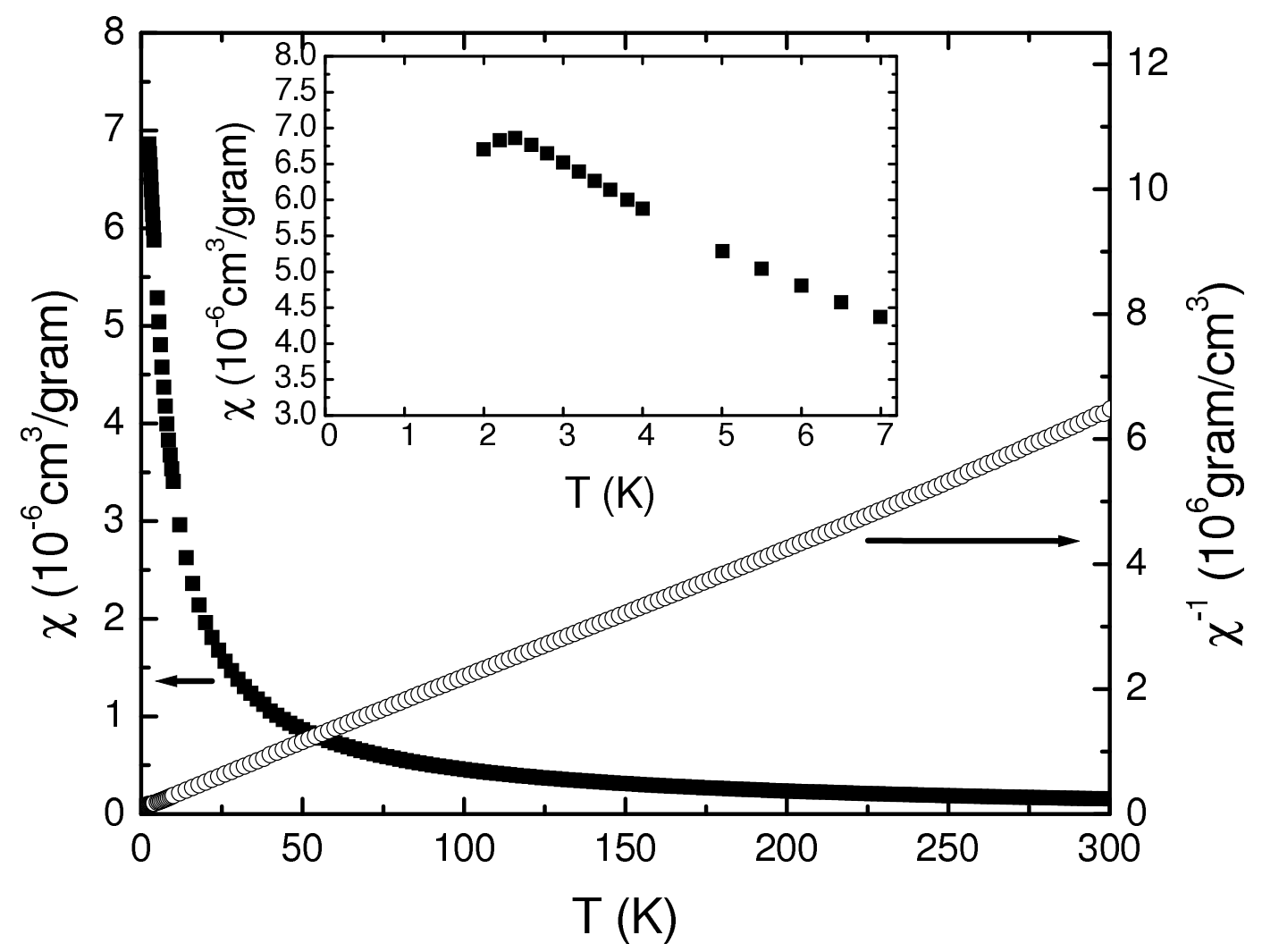

Fig. 1. Magnetic gram-susceptibility (right) and reciprocal gram-susceptibility (left) of our YbGaGe sample. The inset is an enlargement of the susceptibility in the low-temperature region.

made in applied fields of 0.1 and $1.0 \mathrm{~T}$ and the differential susceptibility was calculated eliminating the effects of any constant offset.

The temperature dependent differential magnetic susceptibility is displayed in Figure 1. It shows a maximum near about $2.3 \mathrm{~K}$ (see inset), and decreases continuously as a function of temperature. Figure 1 also shows the temperature dependent reciprocal susceptibility. Above $\sim 5 \mathrm{~K}$, it appears as a nearly straight line, with a slope of about $21 \times 10^{3} \mathrm{~g} \mathrm{~cm}^{-3} \mathrm{~K}^{-1}$.

The maximum in susceptibility near $2.3 \mathrm{~K}$ is due to the presence of $\mathrm{Yb}_{2} \mathrm{O}_{3}$ in the sample, which has been reported to order antiferromagnetically at that temperature (See e.g. Ref.[5]). In this framework, the Curie-Weiss behavior of the reciprocal susceptibility determined for this sample may be interpreted as being solely due to about $0.4 \mathrm{wt} \%$ of an $\mathrm{Yb}_{2} \mathrm{O}_{3}$ impurity present in the sample. This level of impurity is well below the detection level for standard Xray diffraction. The origin of the oxygen in our sample is very likely associated with the intermediate grinding of the sample, in the form of adsorbed gas. It will be shown below that this is consistent with elemental analysis and neutron diffraction. 


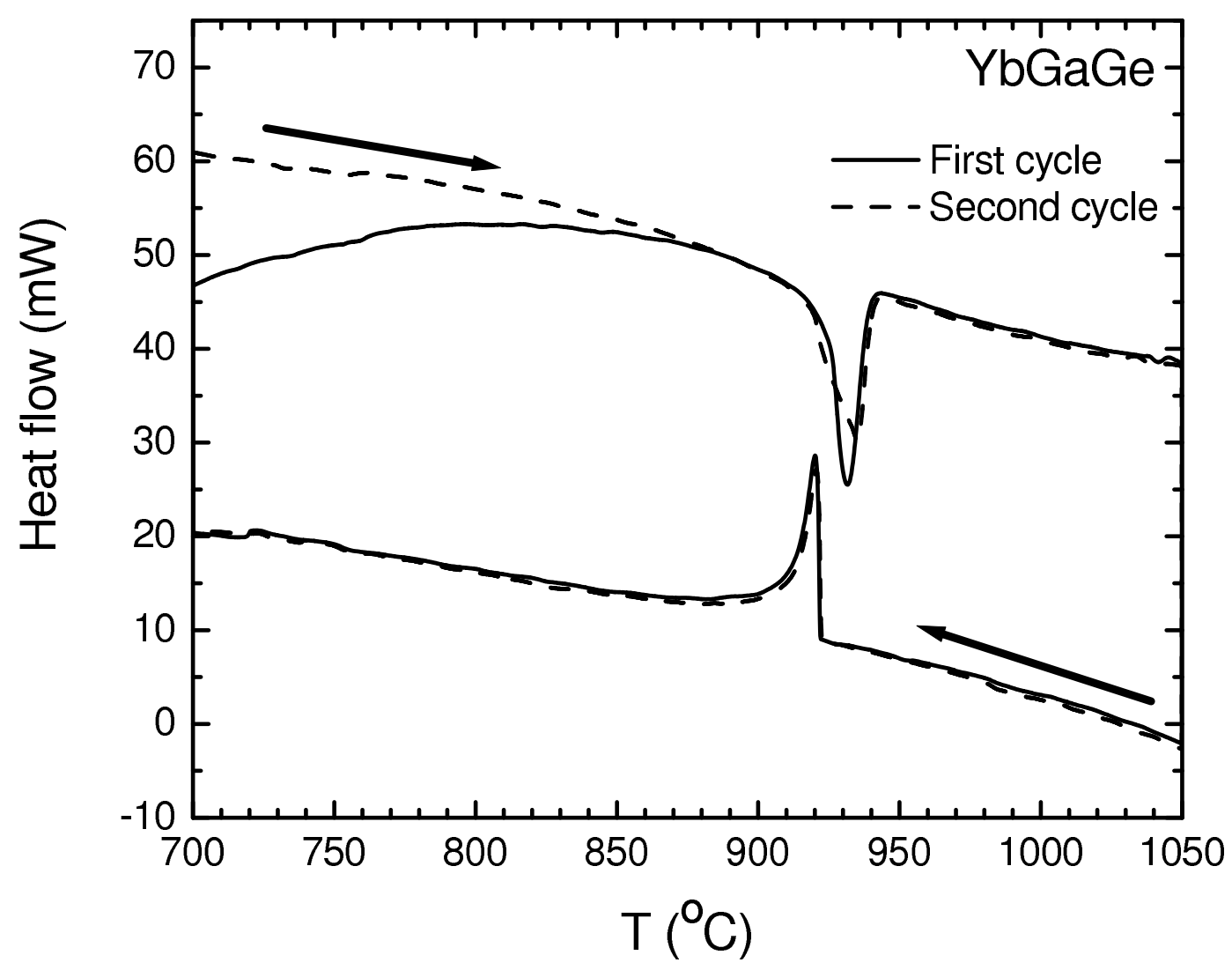

Fig. 2. Relevant part of the DTA trace of our sample of YbGaGe measured upon heating and cooling with a $10^{\circ} \mathrm{C} / \mathrm{min}$ rate. The first heating/cooling cycle is indicated by a full line, the second by a dashed line.

Our results are inconsistent with those reported by Salvador et al.[1]. They are consistent with those reported by Bobev et al. [6], who ascribe the susceptibility of their sample to be mainly due to an impurity phase, and by Muro et al. [7], who observed a weak temperature-independent diamagnetic signal (about $-4.6 \cdot 10^{-5} \mathrm{emu} / \mathrm{mol}$ ) from their sample. It should be noted, that our sample does not show a temperature-independent susceptibility; an estimate of a temperature-independent term of the susceptibility, $\chi_{0}$, from a plot (not shown here) of $\chi$ vs. $1 / T$ in the high-temperature limit yielded a value that is indistinguishable from zero.

Atomic emission spectroscopy on our sample was performed by the commercial laboratory NSL Analytical Services Inc. The Yb-Ga-Ge ratio was found to be stoichiometric to within the experimental uncertainties of the analysis (on average $2 \%$ for each element). A small amount of $\mathrm{O}$ was identified in our sample. Assuming that all the $\mathrm{O}$ atoms belong to an $\mathrm{Yb}_{2} \mathrm{O}_{3}$ impurity, we can assign approximately $0.59(8)$ wt.\% of our sample to the impurity phase, consistent with our magnetization results.

Differential thermal analysis (DTA) was performed in a PerkinElmer Pyris DTA 7 differential thermal analyzer. Our instrument has been set up to pro- 
vide an extremely low partial pressure of oxygen during the measurement. The partial pressure of oxygen of the Zr-gettered ultra-high-purity Ar process gas was monitored at the output of the DTA furnace, and was less than 250 ppm during the measurement. Initial measurements performed in an $\mathrm{Al}_{2} \mathrm{O}_{3}$ crucible showed clear evidence that the sample, after melting, reacted with the crucible. In order to avoid this problem, a small-diameter Ta tube was used to fabricate a crucible, that was put inside the standard $\mathrm{Al}_{2} \mathrm{O}_{3}$ crucible. Figure 2 displays the DTA results obtained using the Ta crucible. The sample was cycled between $600^{\circ} \mathrm{C}$ to $1160^{\circ} \mathrm{C}$ twice, at a rate of $10^{\circ} \mathrm{C} / \mathrm{min}$. Upon heating, a sharp endotherm with an onset temperature of about $920^{\circ} \mathrm{C}$ is observed. No further thermal events were observed up to $1160^{\circ} \mathrm{C}$. Upon cooling, a corresponding exotherm is observed. This reproducible behavior indicates that we have eliminated both a possible oxidation due to the process gas and a reaction with the crucible. Furthermore, our results are consistent with YbGaGe congruently melting around $920^{\circ} \mathrm{C}$. This hypothesis was verified by heating appropriate amounts of the constituent elements up to $1190^{\circ} \mathrm{C}$ in a sealed Ta crucible and subsequently cooling to room temperature in about 12 hours. Powder X-ray diffraction indicated this sample to be single phase YbGaGe.

The results of our differential thermal analysis are inconsistent with those reported by Salvador et al. [1], who saw no melting or phase change up to $1000^{\circ} \mathrm{C}$. The congruent melting is consistent with Bridgman growth out of a stoichiometric melt as reported by Muro et al [7].

\section{Neutron diffraction}

Time-of-flight neutron powder diffraction data were collected at 12 different temperatures between $20 \mathrm{~K}$ and $300 \mathrm{~K}$ for about 3 hours at each temperature on the High Intensity Powder Diffractometer at the Los Alamos Neutron Science Center. The experiments were performed on $3.20 \mathrm{~g}$ of powdered YbGaGe sealed in a vanadium can. Temperature control was provided by a closed-cycle He refrigerator. Data from the $\pm 153^{\circ}$ banks were simultaneously analyzed with the Rietveld refinement package GSAS [8].

Analysis of our neutron-powder-diffraction data confirmed that our sample contains a majority of well crystallized YbGaGe phase and about $0.5 \mathrm{wt} \%$ of $\mathrm{Yb}_{2} \mathrm{O}_{3}$. This is consistent with our atomic emission spectroscopy results, and provides further justification for our approach to understanding the susceptibility of our sample.

The Bragg peaks from the main phase were consistent with the hexagonal structure (spacegroup $P 6_{3} / m m c$, number 194) reported in Ref. [1] for $\mathrm{Yb}$ - 
Table 1

Refined structural parameters of YbGaGe at $20 \mathrm{~K}$. For this fit $\chi^{2}=4.778$, and the weighted residual value $R_{w p}=2.47 \%$.

\begin{tabular}{cccccc}
\hline \hline atom & site & occupation & $\mathrm{x}$ & $\mathrm{y}$ & $\mathrm{z}$ \\
\hline $\mathrm{Yb}_{1}$ & $2 a$ & 1 & 0 & 0 & 0 \\
$\mathrm{Yb}_{2}$ & $2 b$ & 1 & 0 & 0 & $1 / 4$ \\
$\mathrm{Ga}$ & $4 f$ & 1 & $1 / 3$ & $2 / 3$ & $0.15403(3)$ \\
$\mathrm{Ge}$ & $4 f$ & 1 & $1 / 3$ & $1 / 3$ & $0.61312(4)$ \\
\hline
\end{tabular}

Spacegroup: $P 6_{3} / m m c$

$a=4.19186(2)$

$c=16.6709(2)$

GaGe. Therefore, in our refinements we have assumed the Yb atoms to occupy the reported $2 a$ and $2 b$ sites, whereas the Ga and Ge atoms occupy two different $4 f$ sites. We used the $20 \mathrm{~K}$ data to test various models which allow different arrangements of the $\mathrm{Ga}$ and $\mathrm{Ge}$ atoms on the two $4 f$ sites, ranging from a totally random distribution to a completely ordered distribution. The best fit to the observed diffraction profiles were given by a model with an ordered distribution of $\mathrm{Ga}$ and Ge atoms, in agreement with the assignments made in Ref. [1]. However, we do find a small improvement in the refinement if we allow a Ga deficiency of about 6.4(6)at\%. The improvement is marginal and inconsistent with the atomic emmission spectroscopy results. The refined structural parameters, as well as the lattice parameters, at $20 \mathrm{~K}$ are shown in Table 1.

Data taken at subsequent temperatures were used to determine the temperature dependence of the lattice parameters. Generally, the lattice parameters we have found at room temperature, compare very well to those reported elsewhere $[1,7,6]$. We find no evidence of an anomalous thermal expansion, as can be seen in Figure 3. In fact, we find that both lattice parameters $a$ and $c$ in our sample increase smoothly with increasing temperature in the range 20 - $300 \mathrm{~K}$ (see inset of Figure 3). In addition, our estimate of the room temperature volume expansion coefficient $\beta(300 K) \approx 4.35 \times 10^{5} \mathrm{~K}^{-1}$ is comparable to other metals such as $\mathrm{Cu}\left(\sim 5 \times 10^{5} \mathrm{~K}^{-1}\right)$ [9]. This is in contrast to the zero thermal expansion reported by Salvador et al. [1]. The normal thermal expansion we have found is in agreement with the work by Muro et al.[7], and by Bobev et al.[6]. 


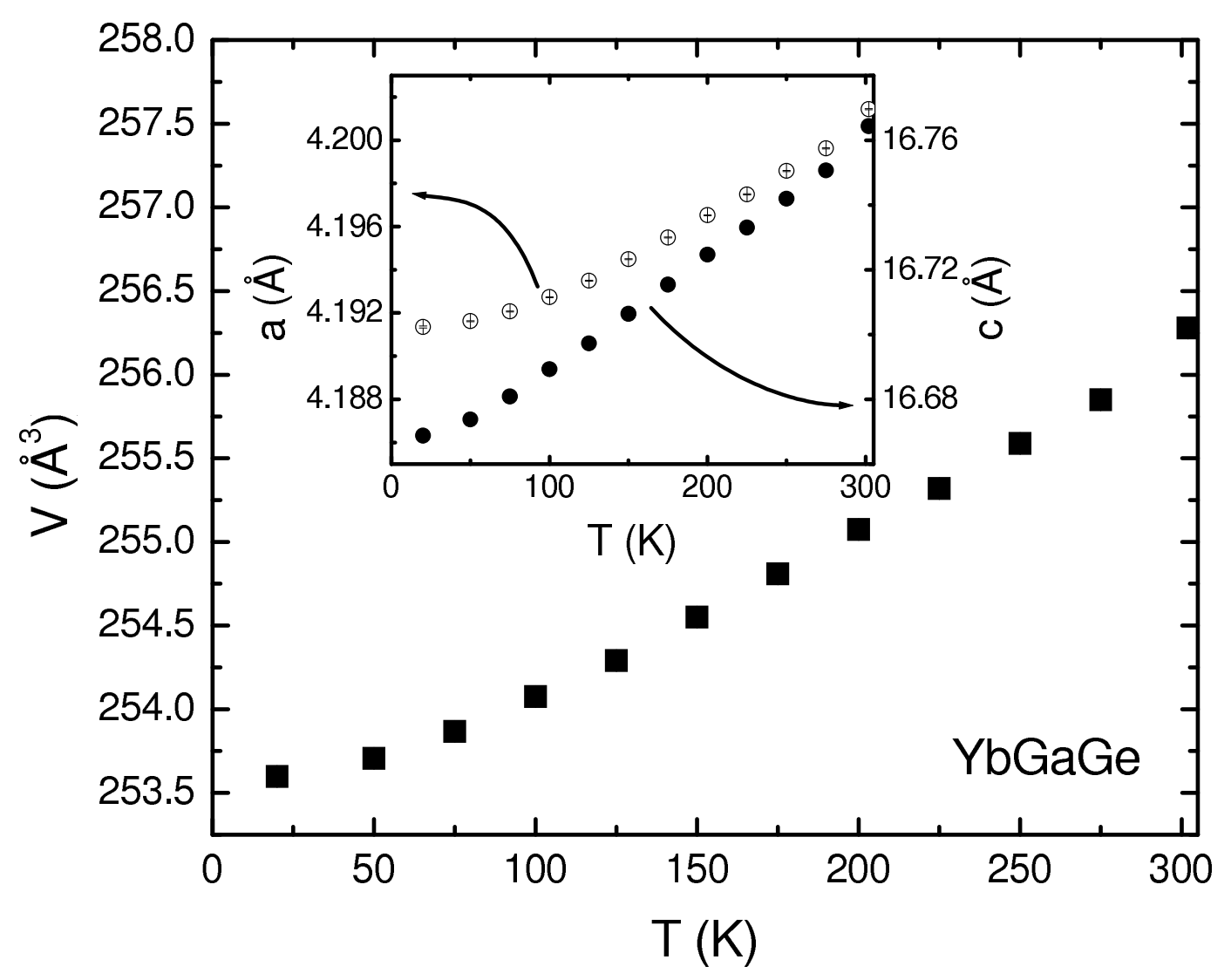

Fig. 3. Unit cell volume of $\mathrm{YbGaGe}$ as a function of temperature obtained from neutron diffraction experiments. The inset shows the the lattice parameters $a$ (open circles) and $c$ (solid circles) as a function of temperature.

\section{Conclusions}

The reported crystal structure of YbGaGe[1], including the site occupations of $\mathrm{Ga}$ and $\mathrm{Ge}$, is confirmed by our neutron diffraction experiments. In agreement with Refs. [6] and [7], zero or negative thermal expansion is not observed in our well-characterized bulk sample of YbGaGe, instead the thermal expansion is comparable to that of $\mathrm{Cu}\left(\sim 5 \times 10^{-5} \mathrm{~K}^{-1}\right)[9]$. Furthermore, from bulk magnetization results there is no evidence for a valence change at temperatures down to $1.8 \mathrm{~K}$, and the small, but non-zero signal is attributed to about 0.5 wt\% of $\mathrm{Yb}_{2} \mathrm{O}_{3}$. The presence of $\mathrm{Yb}_{2} \mathrm{O}_{3}$ in our sample was confirmed by neutron diffraction.

In addition to a normal thermal expansion, differential thermal analysis performed in an atmosphere with an extremely low partial pressure of oxygen, and using an inert Ta crucible, indicated our sample of YbGaGe to melt congruently at about $920^{\circ} \mathrm{C}$. This is in sharp contrast to results reported in Ref. [1]. The elemental analysis reported by Salvador et al. and the atomic emission spectroscopy on our sample agree very well. Since the liquidus should not have 
a discontinuous peak at the melting point of a compound [10], samples with similar composition should have similar melting temperatures. Therefore, we find it difficult to reconcile our differential thermal analysis with the analysis reported, since both samples have a very similar composition, but behave very differently when heated.

We think that the differences between our results and those of Salvador et al. may be related to different purities of the starting elements or due to different crucible materials.

\section{Acknowledgments}

The authors wish to thank M. Angst and S.L. Bud'ko for their invaluable input during the preparation of this manuscript, and A.C. Lawson, J. Zarestky and V.O. Garlea for their assistance with neutron data. Ames Laboratory is operated for the US Department of Energy by Iowa State University under Contract No. W-7405-Eng-82. This work has benefited from the use of the Los Alamos Neutron Science Center at the Los Alamos National Laboratory. This facility is funded by the US Department of Energy under Contract W-7405Eng-36. This work was supported by the Director for Energy Research, Office of Basic Energy Sciences.

\section{References}

[1] J. R. Salvador, F. Guo, T. Hogan, and M.G. Kanatzidis, Nature 425, 702 (2003), and J. R. Salvador, F. Guo, T. Hogan, and M.G. Kanatzidis, Nature 426, 584 (2003).

[2] S. Margadonna, K. Prassides, A.N. Fitch, J.R. Salvador, and M. G. Kanatzidis, J. Am. Chem. Soc. 126, 4498 (2004).

[3] A. W. Sleight, Inorg. Chem. 37, 2854 (1998).

[4] A. C. Lawson, J. A. Roberts, B. Martinez, J. W. Richardson, Jr., Phil. Mag. B 82, 1837 (2002); S. Y. Savrasov, G. Kotliar, E. Abrahams, Nature (London) 410, 793 (2002).

[5] H. Li, C.Y. Wu, and J.C. Ho, Phys. Rev. B 49, 1447 (1994).

[6] S. Bobev, D.J. Williams, J.D. Thompson, and J.L. Sarrao, Solid State Commun., in print (cond-mat0405063)

[7] Y. Muro, T. Nakagawa, K. Umeo, M. Itoh, T. Suzuki, and T. Takabatake, J. Phys. Soc. Jpn, in print 
[8] A. C. Larson and R. B. Von Dreele, GSAS - General Structure Analysis System, Los Alamos National Laboratory Report No. LA-UR-86-748.

[9] F. C. Nix and D. MacNair, Phys. Rev. 60, 597 (1941)

[10] H. Okamoto and T.B. Massalski, J. Phase Equilibiria 12, 148 (1991) 